\title{
ON THE RANGE OF COMPLETELY BOUNDED MAPS
}

\author{
RICHARD I. LOEAL \\ Department of Mathematics \\ Wayne State University \\ Detroit, Michigan 48202 \\ (Received March 28, 1977)
}

ABSTRACT. It is shown that if every bounded linear map from a $c^{\star}$-algebra $a$ to a von Neumann algebra $B$ is completely bounded, then either $a$ is finitedimensional or $B \leq C \otimes M_{n}$, where $C$ is a commutative von Neumann algebra and $M_{n}$ is the algebra of $n \times n$ complex matrices.

Let $a, \beta$ be $c^{*}$-algebras, and let $\varphi: a \rightarrow \beta$ be a bounded linear map. For every positive integer $n$, we define the map $\varphi_{n}$ to be $\varphi_{n}=\varphi \otimes 1 d_{n}$, the entry-wise map from $a \otimes M_{n}$ to $B \otimes M_{n}$, where $M_{n}$ denotes the $C^{*}$-algebra of $n \times n$ complex matrices. We say that $\varphi$ is completely bounded if $\sup _{n}\left\|\varphi_{n}\right\|<\infty[1]$. It is not a priori evident that there are bounded maps which fail to be completely bounded. It follows from the results of this paper that there are almost always such maps. 
Let us denote by $B_{1}[a, B]$ the set of all bounded maps from $a$ to $B$, and by $B_{\infty}[a, B]$ the set of all completely bounded maps from $a$ to $B$. We shall describe some of the structure of $B_{\infty}[a, B]$ below. Further, in a previous paper [3], we made the following Conjectures:

(1) If $B_{1}[a, B]=B_{\infty}[a, B]$ for all $a$, then $B \subseteq C \otimes M_{n}$ for some commutative $C^{*}$-algebra $C$ and integer $n$;

(2) If $B_{1}[a, \beta]=B_{\omega}[a, B]$, then either $a$ is finite-dimensional or $B=C_{n} \otimes M_{n}$

We shall give an affirmative answer to both these conjectures under the hypothesis that $\beta$ is a von Neumann algebra $R$. We should remark that the converses to (1) and (2) hold; i.e., if $a$ is finite-dimensional or $B \subseteq C \otimes M_{n}$, then $B_{1}[Q, B]=B_{\infty}[a, B]$ (see below).

Although our proof depends heavily on the hypothesis that the range is a von Neumann algebra, we feel that this is merely a shortcoming of our proof, and not a true reflection of the facts.

We begin with what is, to the best of our knowledge, the only example in the literature of a bounded map that is not completely bounded.

THEOREM 1: Let $\mathrm{X}$ be an infinite compact Hausdorff space. Then there Is a bounded map $\varphi: C(X) \rightarrow \oplus_{n} M_{2} n$ such that $\varphi$ is not completely bounded. Further, if $c(x) \subseteq a$, where $a$ is a $c^{*}$-algebra, then $\varphi$ has an extension $\tilde{\varphi}$ to a such that $\left\|\tilde{\varphi}_{n}\right\|=\left\|\varphi_{n}\right\|$ for all $n$.

PROOF: The proof of the first assertion can be found in [4, Lemma 2.1 and Theorem 2.2], and the second assertion follows from the construction used to produce $\varphi$. We will sketch the highlights of the construction, both for the convenience of the reader and for later reference.

Let $C=C(X)$. For every integer $n$, there exist elements $A_{1}, \ldots, A_{n} \in M_{2} n$ such that: (1) $A_{1}=A_{i}^{*}$; (2) $A_{i} A_{j}+A_{j} A_{i}=2 b_{i j} I n$; and (3) $\operatorname{Tr}\left(A_{1}\right)=0$. 
Let $\rho_{1}^{(n)}, \ldots, \rho_{n}^{(n)}$ be positive linear functionals on $C$ with disjoint closed supports and let $\varphi^{(n)}(f)=\sum_{i=1}^{n} \rho_{i}^{(n)}(f) A_{1}$. Then $\left\|\varphi{ }^{(n)}\right\| \leq \sqrt{2} \sqrt{ } \sum\left\|_{\rho}^{(n)}\right\|^{2}$, ,o that if $\left\|\rho_{i}^{(n)}\right\|=n^{-3 / 4}$ for all $1,\|\varphi(n)\| \leq \sqrt{2} n^{-1 / 4}$. We now remark that by Krein's Theorem [5, p. 227], the $\rho_{1}^{(n)}$ have norm-preserving positive extensions to $a>c$, and the extension assertion rests on our demonstrating that the $\left\|\rho{ }_{i}^{(n)}\right\|$ are the keys to computing $\sup _{k}\left\|\varphi_{k}^{(n)}\right\|$.

It is true that for a positive 1inear functional $\tau, \sup _{\mathbf{k}}\left\|\tau_{k}\right\|=\|\tau\|$ [1, Prop. 1.2.10]. Thus, $\sup _{k}\left\|\varphi_{k}^{(n)}\right\| \leq \sum_{i=1}^{n}\left\|_{\rho}^{(n)}\right\|=n^{1 / 4}$. But in fact, $\left\|\varphi 2^{(n)}\right\|=\Sigma\left\|_{\rho}^{(n)}\right\|=n^{1 / 4}$.

Let $\psi=\oplus_{\mathrm{n}} \varphi^{(\mathrm{n})}$ where all the functionals $\rho_{1}^{(\mathrm{n})}$ are chosen to have disjoint closed supports, which is possible since $X$ is infinite. Then $\|\psi\|=\sup _{n}\|\varphi(n)\| \leq \sqrt{2} \sup _{n} n^{-1 / 4}=\sqrt{n}$. But $\sup _{k}\left\|\psi_{k}\right\|=\sup _{n, k}\left\|\varphi_{k}^{(n)}\right\| \geq \sup _{n}\left\|\varphi_{2}^{(n)}\right\|=$ $\sup _{n} n^{1 / 4}=+\infty$, and thus fails to be completely bounded.

COROLLARY 2: If $\mathcal{N}$ is an infinite dimensional Hilbert space, and $\mathrm{X}$ is an infinite compact Hausdorff space, there is a bounded map $\varphi: C(X) \rightarrow \mathcal{L}(\aleph)$, the algebra of all bounded operators on $\mathcal{N}$, such that $\varphi$ is not completely bounded.

If $\varphi: a \rightarrow B$ is a linear map of $c^{*}$-algebras, we define the adjoint of $\varphi, \varphi^{*}$, by $\varphi^{*}(\mathrm{~A})=\varphi\left(\mathrm{A}^{*}\right)^{*}$. Then $\varphi^{*}$ is a linear map from $a$ to $B$, and $\|\varphi *\|=\|\varphi\|$. We say that $\varphi$ is self-adjoint if $\varphi=\varphi^{*}$. Every map $\varphi$ can be written uniquely as $\varphi=\varphi_{1}+i \varphi_{2}$, where $\varphi_{1}, \varphi_{2}$ are self-adjoint.

PROPOSITION 3: $\quad B_{\infty}[a, \beta]$ is a self-adjoint linear subspace of $B_{1}[a, \beta]$, but $B_{\infty}$ need not be norm-closed.

PROOF: It is elementary that for all $k,\left\|\varphi_{k}\right\|=\left\|\varphi_{k}^{*}\right\|$, and that the sum or scalar multiples of completely bounded maps are completely bounded. For the second assertion, let us re-examine the proof of Theorem 1 . Let $\psi^{(N)}=\oplus_{n=1}^{N} \varphi^{(n)}$; then $\left\|\psi-\psi^{(N)}\right\|=\sup _{n>N}\|\varphi(n)\| \leq \sqrt{2}(N+1)^{-1 / 4} \rightarrow 0$ as $N \rightarrow \infty$, 
so $\psi^{(\mathrm{N})} \rightarrow \varphi$. However, $\sup _{k}\left\|\psi_{k}^{(N)}\right\|=(\mathrm{N})^{1 / 4}$, so each $\psi^{(\mathrm{n})}$ is completely bounded.

We remark that if one defines $\|\varphi\|=\sup _{k}\left\|\varphi_{k}\right\|$, then $B_{\infty}[a, B]$ is closed in ||$|\cdot| \mid$. We suspect that $B_{\infty}[a, B]$ is always dense in $B_{1}[a, B]$, at least in the weak (i.e., pointwise) topology.

We also should remark that if $\theta$ is a $*$-isomorphism of $C^{*}$-algebras, then $\|\varphi \circ \theta\|=\|\theta \circ \varphi\|=\|\varphi\|$ and more generally, $\left\|(\varphi \circ \theta)_{k}\right\|=\left\|(\theta \circ \varphi)_{k}\right\|=\left\|\varphi_{k}\right\|$ for all integers $k$. Thus the classes $B_{1}[a, \beta]$ and $B_{\infty}[a, \beta]$ are essentially unchanged under isomorphism; e.g., if $a \cong a_{1}$ and $\beta \cong \beta_{1}$, then $B_{\infty}[a, \beta] \cong$ $\mathrm{B}_{\infty}\left[a_{1}, B_{1}\right]$

We will denote by $M_{\infty}$ the algebra $\mathcal{L}(\mathcal{K})$, where $\mathcal{N}$ is a separable infinite-dimensional Hilbert space. By Corollary $2, M_{\infty} 2 \oplus_{n} M_{2} n \cdot$

We will now do an analysis of von Neumann algebras, based on their type, that will identify the characteristics we need. We follow the type classification of [6, pp. 24-25]

LEMMA 4: Let $R$ be a von Neumann algebra of type $I_{\infty}$, II $I_{\infty}$ or III. Then $R 2$ (an isomorphic copy of) $M_{\infty}$.

PROOF: By [6, Cor. 14], $R$ is spatially isomorphic to $R \otimes M_{\infty}$, but $R \otimes M_{\infty} 21 \otimes M_{\infty} \cong M_{\infty}$.

LEMMA 5: Let $R$ be a von Neumann algebra of type II $_{1}$. Then $R 2$ (an isomorphic copy of) $\underset{n}{\oplus} 2_{2}$.

PROOF: Let $\left\{P_{n}\right\}_{n=1}^{\infty}$ be family of non-zero, orthogonal projections in $R[6, p .45]$. For each $n$, write $P_{n}=\bigoplus_{i=1}^{2^{n}} E_{i}^{(n)}$, where $E_{i}^{(n)} \perp E_{j}^{(n)}$ for $1 \neq j$, and $E_{i}^{(n)}$ is equivalent to $E_{j}^{(n)}$ in the usual sense of equivalence for projections. Let $\left\{v_{i j}^{(n)}\right\}$ be partial isometries (in $P_{n} R P_{n}$ [P. 46, Remark]) such that $v_{i j}^{(n) *} v_{i j}^{(n)}=E_{j}^{(n)}, v_{i j}^{(n)} v_{i j}^{(n) *}=E_{i}^{(n)}$, so that we can take $v_{j i}^{(n)}=v_{i j}^{(n) *}$ 
Then $\left\{v_{i j}^{(n)}\right\} \cup\left\{E_{i}^{(n)}\right\}$ generate an isomorphic copy of $M_{2} n$. Thus $R 2 \underset{\mathbf{n}}{\oplus} \mathrm{P}_{\mathbf{n}} R_{\mathrm{P}} \geq \underset{\mathrm{n}}{\oplus} \mathrm{M}_{2} \mathrm{n}$

LEMMA 6: Let $R=\underset{\alpha}{\oplus} R_{\alpha}$, where $R_{\alpha}$ is a homogeneous von Neumann algebra of degree $I_{n_{\alpha}}, 1 \leq n_{\alpha}<\infty$. If $\sup _{\alpha} n_{\alpha}=\infty$, then $R 2$ (an isomorphic copy of) $\bigoplus_{n} M_{2} n$.

PROOF: Each $R_{\alpha}$ is (isomorphically) of the form $C_{\alpha} \otimes M_{n_{\alpha}}$, where $c_{\alpha}$ is a commutative von Neumann algebra [6, p. 98], and thus $R_{\alpha} 21 \otimes M_{n_{\alpha}} \cong M_{n_{\alpha}}$. If $\sup _{\alpha} n_{\alpha}=\infty$, there is a subsequence $n_{\alpha_{i}}$ such that $n_{\alpha_{1}} \geq 2^{i}$, and thus $R_{\alpha_{1}} 2 M_{n_{\alpha_{1}}} 2 M_{2}$. Then $R 2 \oplus R_{\alpha_{1}} 2 \underset{i}{\oplus} M_{2}$.

We are now ready to prove the main result of this paper.

THEOREM 7. Let $R$ be a von Neumann algebra, and suppose that for some Infinite compact Hausdorff space $X, B_{1}[C(X), R]=B_{\infty}[C(X), R]$. Then there is a commutative $C^{*}$-algebra $C$ and integer $n$ such that $R \subseteq C \otimes M_{n}$.

PROOF: We can write $R$ as a (unique) direct sum $R=R_{1} \oplus R_{2} \oplus R_{3} \oplus R_{4}$ $\oplus R_{5}$ where $R_{1}$ is of type $I_{\infty}, R_{2}$ of type $I_{\infty}, R_{3}$ of type III, $R_{4}$ of type II 1 , and $R_{5}$ of type $I_{n}[6, p .25]$. By using Theorem 1, Corollary 2, Lemma 4, and Lemma 5 , we see that the hypothesis forces $R_{1}=R_{2}=R_{3}=R_{4}=0$. Thus $R=R_{5}$ is of type $I_{n}$, so $R=\underset{\alpha}{\oplus} R_{\alpha}$, where $R_{\alpha}$ is homogeneous of degree $n_{\alpha}[6, p, 42]$. By applying Theorem 1, Lemma 6, and the hypothesis, we see that $\sup _{\alpha} n_{\alpha}=N<\infty$. But then $R=\underset{\alpha}{\oplus}\left(C\left(X_{\alpha}\right) \otimes M_{n_{\alpha}}\right) \subseteq \underset{\alpha}{\oplus}\left(C\left(X_{\alpha}\right) \otimes M_{n}\right) \subseteq$ $C \otimes M_{N}$, for an appropriate commutative $c^{*}$-algebra $c$.

We can now give our answer to the first conjecture.

COROLLARY 8: If $R$ is a von Neumann.algebra, and for all $c^{*}$-algebras a, $B_{1}[a, R]=B_{\infty}[a, R]$, then $R \subseteq C \otimes M_{n}$.

For the sake of completeness, we state a converse to Theorem 7. The proof may be found in [3, Lemma 7]. 
PROPOSITION 9: If $B \subseteq C \otimes M_{n}$, then for all $C^{*}$-algebras $a$, $\mathrm{B}_{1}[a, \beta]=\mathrm{B}_{\infty}[a, \beta]$.

In order to present our answer to the second conjecture, we need the following result.

PROPOSITION 10: Let $a$ be an infinite-dimensional $c^{*}$-algebra. Then $a \geq c(x)$ for some infinite compact Hausdorff space $x$.

PROOF: This fact is established in the proof of [3, Thm. E], and is a variation upon [2]

THEOREM 11: If $R$ is a von Neumann algebra, and for some $C^{*}$-algebra $a, B_{1}[a, R]=B_{\infty}[a, R]$, then either $a$ is finite-dimensional or $R 2 \mathrm{C} \otimes M_{n}$.

PROOF: The proof of Theorem 7 shows that either $R \subseteq C \otimes M_{n}$ or $R 2 \underset{n}{\oplus} M_{2} n$. Suppose the latter; then if $a$ is infinite-dimensional, we see from Proposition 10 that $a \geq \mathrm{C}(\mathrm{X})$ for some infinite compact Hausdorff space $\mathrm{x}$. But then by Theorem 1 , there is a map $\varphi, \varphi: a \rightarrow \oplus_{\mathrm{n}} M_{2} \mathrm{n} \in R$, which is bounded but not completely bounded. This contradicts the hypothesis, and completes the proof.

For the sake of completeness, we state a result, which along with Proposition 9, yields a converse to Theorem 11. The proof may be found in [3, Lemma 5].

PROPOSITION 12: If $a$ is finite-dimensional, then for all $c^{*}$-algebras $B, \quad B_{1}[A, B]=B_{\infty}[A, B]$.

We remark that, by Theorem 11 , there are almost always bounded maps between $\mathrm{C}^{*}$-algebras that fail to be completely bounded.

We should mention that there is another interesting consequence of the methods of this paper.

THEOREM 13. Let $a$. be an infinite-dimensional $c^{*}-a 1 g e b r a$. Then there is a bounded self-adjoint map $\varphi: a \rightarrow \underset{\mathrm{n}}{\oplus} \mathrm{M}_{2} \mathrm{n}$ such that $\varphi$ cannot be written $\varphi=\varphi^{+}-\varphi^{-}$where $\varphi^{+}, \varphi^{-}$are bounded positive linear maps from $a \rightarrow \mathcal{L}(\mathcal{*})$. 
PROOF: The actual assertion of [4, Lemma 2.1 and Theorem 2.2] is that the map $\varphi: C(X) \rightarrow \underset{n}{\oplus} M_{2} n$ described in the proof of Theorem 1 does not admit such a decomposition. The extension statement in Theorem 1 and Proposition 10 allow us to pass to an arbitrary infinite-dimensional $a$.

Arguments similar to those of Lemmas 4, 5, 6 and Theorem 7 in this paper were used in the dissertation of Sze-Kai Jack Tsui, University of Pennsylvania, 1975. He also obtained results relating to Theorem 13 of this paper.

\section{BIBLIOGRAPHY}

1. Arveson, W. B. Subalgebras of $C^{*}$-algebras, Acta Math. 123 (1969), 141-224.

2. Hirschfeld, R. A. and Johnson, B. E. Spectral characterization of finitedimensional algebras, Indag. Math. 34 (1972), 19-23.

3. Loeb1, R. I. Contractive linear maps on $C^{*}$-algebras, Michigan Math. J. 22 (1975), 361-366.

4. A Hahn decomposition for linear maps, Pacific J. Math., 65 (1976), 119-133.

5. Rickart, C. E. General Theory of Banach Algebras, Van Nostrand, New York, 1960.

6. Topping, D. M. Lectures on von Neumann Algebras, Van Nostrand Reinhold, London, 1971 .

KEY WORDS AND PHRASES. Completely bounded maps, $C^{*}$-algebras, von Neumann algebras, extension of maps. 


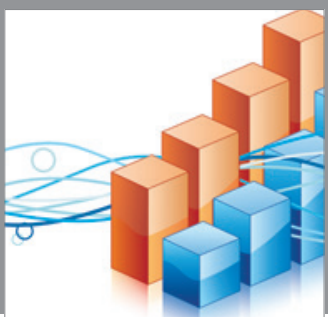

Advances in

Operations Research

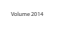



\section{The Scientific} World Journal
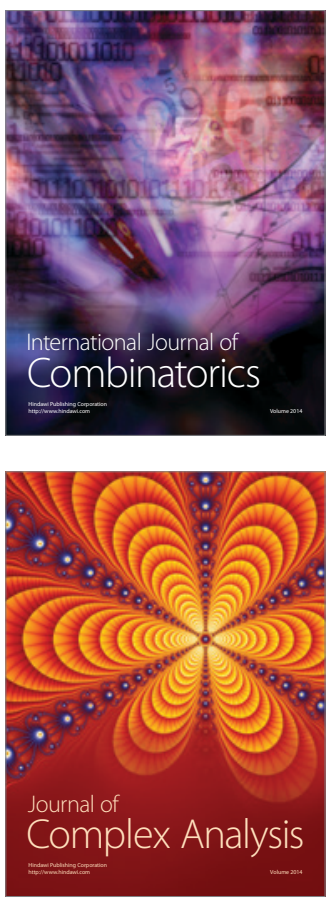

International Journal of

Mathematics and

Mathematical

Sciences


Journal of

Applied Mathematics
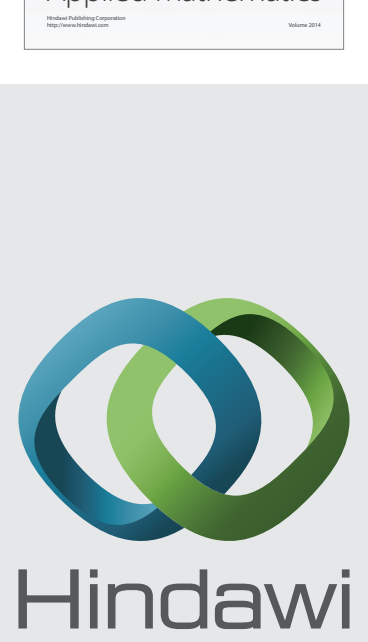

Submit your manuscripts at http://www.hindawi.com
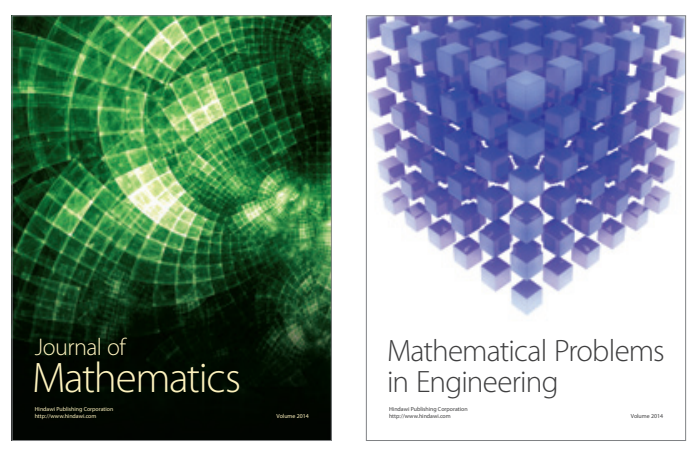

Mathematical Problems in Engineering
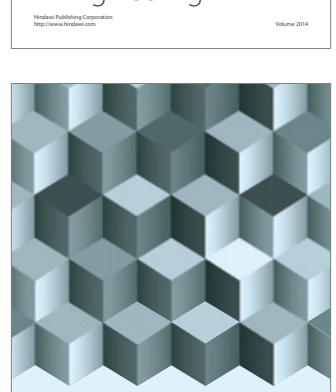

Journal of

Function Spaces
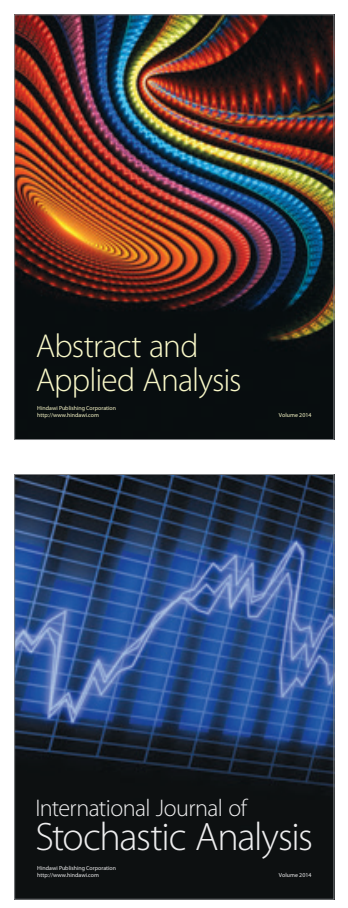

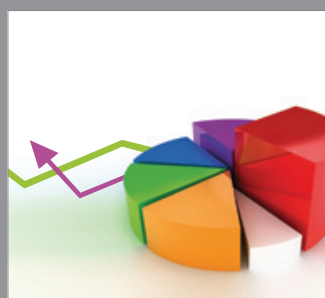

ournal of

Probability and Statistics

Promensencen
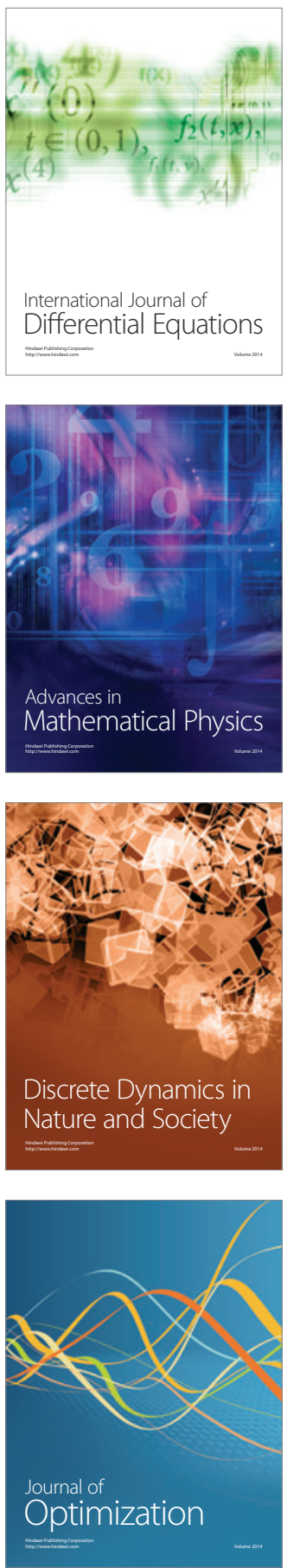